\title{
Opioids for Back Pain Patients: Primary Care Prescribing Patterns and Use of Services
}

\author{
Richard A. Deyo, MD, MPH, David H. M. Smith, RPh, PhD, Eric S. Johnson, PhD, \\ Marilee Donovan, RN, PhD, Carrie J. Tillotson, MPH, Xiuhai Yang, MS, \\ Amanda F. Petrik, MS, and Steven K. Dobscha, MD
}

Background: Opioid prescribing for noncancer pain has increased dramatically. We examined whether the prevalence of unhealthy lifestyles, psychologic distress, health care utilization, and co-prescribing of sedative-hypnotics increased with increasing duration of prescription opioid use.

Methods: We analyzed electronic data for 6 months before and after an index visit for back pain in a managed care plan. Use of opioids was characterized as "none," "acute" ( $\leq 90$ days), "episodic," or "long term." Associations with lifestyle factors, psychologic distress, and utilization were adjusted for demographics and comorbidity.

Results: There were 26,014 eligible patients. Of these, $61 \%$ received a course of opioids, and 19\% were long-term users. Psychologic distress, unhealthy lifestyles, and utilization were associated incrementally with duration of opioid prescription, not just with chronic use. Among long-term opioid users, 59\% received only short-acting drugs; $39 \%$ received both long- and short-acting drugs; and $44 \%$ received a sedative-hypnotic. Of those with any opioid use, $36 \%$ had an emergency visit.

Conclusions: Prescription of opioids was common among patients with back pain. The prevalence of psychologic distress, unhealthy lifestyles, and health care utilization increased incrementally with duration of use. Coprescribing sedative-hypnotics was common. These data may help in predicting long-term opioid use and improving the safety of opioid prescribing. (J Am Board Fam Med 2011;24:717-727.)

Keywords: Analgesics, Back Pain, Pain Control, Quality of Health Care

Chronic pain is a common complaint in primary care. More than $2 \%$ of US adults report regular use of prescription opioids, and more than half of these have chronic back pain. ${ }^{1}$ Many such patients have persistent high levels of pain and poor quality of life despite opioid therapy. ${ }^{2}$ Despite uncertainties about long-term efficacy and safety for chronic back pain, prescription opioid use has increased rapidly, and complications related to overdoses and diversion have risen in parallel. ${ }^{3-11}$

This article was externally peer reviewed.

Submitted 8 October 2010; revised 1 April 2011; accepted 6 April 2011.

From the Department of Family Medicine (RAD), the Department of Medicine (RAD), and the Department of Psychiatry (SKD), the Oregon Clinical and Translational Research Institute (RAD, CJT), and the Center for Research in Occupational and Environmental Toxicology (RAD), Oregon Health and Science University; the Kaiser Permanente Center for Health Research (RAD, DHMS, ESJ, MD, XY, AP); and the Center for Study of Comorbid Mental and Physical Disorders, Portland Veterans Affairs Medical Center (SKD), Portland, OR.
Patients with anxiety or depression are more likely than others to have opioids prescribed yet have less analgesic benefit, are more prone to medication misuse, and are more likely to use psychoactive drugs. ${ }^{12-14}$ Whether psychologic distress is associated with acute opioid use as opposed to chronic use is unclear. A few studies have suggested that lifestyle factors such as smoking and obesity are predictors of opioid misuse or early opioid prescriptions for pain, but these associations have not been well studied in routine clinical care. ${ }^{15,16}$

Funding: This study was made possible with support from the Oregon Clinical and Translational Research Institute grant no. UL1 RR024140; from the National Center for Research Resources, a component of the National Institutes of Health; and the National Institutes of Health Roadmap for Medical Research.

Conflict of interest: none declared.

Corresponding author: Richard A. Deyo, Department of Family Medicine, Mail Code FM, Oregon Health and Science University, 3181 SW Sam Jackson Park Road, Portland, OR 972329 (E-mail: deyor@ohsu.edu). 
Certain opioid prescribing patterns raise important safety concerns. For example, co-prescribing opioids and benzodiazepines is recognized as a risk for unintentional overdose and death, ${ }^{6,17}$ but the frequency of such prescribing in routine care is not well characterized.

We used electronic health records for primary care patients with chronic back pain in a large managed care plan to examine patterns of opioid use. Our goals were to clarify the nature of associations between mental health, lifestyle, and opioid prescribing and to identify potential strategies for improving the efficiency and safety of opioid prescribing in primary care. Our study aims were:

1. To examine patient and prescribing characteristics associated with long-term opioid use. We hypothesized that diagnoses of substance abuse, depression, and anxiety would be associated with higher rates of long-term opioid use. We also hypothesized that lifestyle factors (obesity, smoking) and greater comorbidity would be associated with greater opioid use. We further sought to determine whether these characteristics were associated only with long-term opioid use or also with acute use, perhaps in an incremental fashion.

2. To examine the prevalence of a patient safety concern: co-prescription of sedative-hypnotics and opioids. We hypothesized that co-prescription of sedative-hypnotics may be common despite cautions against this practice.

3. To describe use of other health care services that may be associated with opioid use. We hypothesized that among all patients with back pain, emergency department use and frequency of clinic visits would be higher among opioid users than nonusers, helping to identify potentially avoidable visits and improve efficiency of care.

Such analyses would help to clarify the likelihood of long-term opioid use among certain patients; determine if risky co-prescribing is an important problem; and suggest strategies for providing more efficient care for an often challenging patient group.

\section{Methods \\ Setting}

We conducted this study in the Kaiser Permanente Northwest (KPNW) health care system in Portland, Oregon. KPNW is a federally qualified, notfor-profit health management organization serving more than 470,000 members in northwest Oregon and southwest Washington. Members are demographically representative of the coverage area and represent approximately $17 \%$ of the area's population. This group-model delivery system provides the entire scope of care for members, who are encouraged to choose a personal physician and return to that physician for medical care. Family practitioners, internists, and pediatricians provide primary care and maintain continuity of treatment.

KPNW's data systems are accessible for research purposes; all members are informed of this as part of their membership agreement, and they can elect to be excluded from all or some research studies. This study was approved by the institutional review boards at the Kaiser Permanente Center for Health Research and Oregon Health and Science University.

KPNW provides prescriptions at a reduced cost, and prescriptions are prepaid for a significant proportion of the population. The automated outpatient pharmacy system records all prescriptions dispensed. Based on a membership survey, an estimated $90 \%$ of prescriptions are filled at a program pharmacy, including those for members without a prepaid drug benefit. For our study, we required that patients have 1 year of continuous membership and drug coverage before the index visit. Because of that coverage requirement, study patients had financial and logistic incentives to use KPNW pharmacies for their prescriptions. Although KPNW has a formulary of recommended medications, physicians may prescribe any marketed drug.

\section{Patients}

We studied adult ambulatory patients aged 18 and older. To select patients with back pain, we chose as an index visit the first visit in 2004 with any one of 32 International Classification of Diseases, Ninth Revision, Clinical Modification diagnoses associated with low back pain (Table 1 ), ${ }^{18}$ and we used electronic pharmacy and medical record data for 1 year before and 6 months after an index visit. 
Table 1. International Classification of Diseases, 9th Revision, Clinical Modification Diagnosis and Procedure Codes Used to Select or Exclude Patients ${ }^{18}$

\begin{tabular}{|c|c|}
\hline Inclusion codes & Back Pain \\
\hline 721.3 & Lumbosacral spondylosis without myelopathy \\
\hline 721.42 & Spondylogenic compression of lumbar spinal cord \\
\hline 722.10 & Displacement of lumbar intervertebral disc without myelopathy \\
\hline 722.32 & Schmorl's nodes, lumbar \\
\hline 722.52 & Degeneration of lumbar or lumbosacral intervertebral disc \\
\hline 722.73 & Intervertebral disc disorder with myelopathy, lumbar \\
\hline 722.83 & Postlaminectomy syndrome, lumbar \\
\hline 722.93 & Other and unspecified disc disorder, lumbar \\
\hline 724.02 & Spinal stenosis, lumbar \\
\hline 724.2 & Lumbago; low back pain \\
\hline 724.3 & Sciatica \\
\hline 724.5 & Backache, unspecified \\
\hline 724.6 & Disorders of sacrum \\
\hline 738.4 & Acquired spondylolisthesis \\
\hline $739.3,739.4$ & Somatic dysfunction, lumbar region or sacral region \\
\hline 756.11 & Spondylolysis, lumbosacral region \\
\hline 756.12 & Spondylolisthesis \\
\hline $805.4,805.6$ & Vertebral fracture without spinal cord injury, closed, lumbar, sacrum, or coccyx \\
\hline $846.0-846.9$ & Sprains and strains of sacroiliac region \\
\hline $847.2,847.3$ & Sprains and strains, lumbar or sacrum \\
\hline \multicolumn{2}{|l|}{ Exclusion codes } \\
\hline $140-239.9$ & Neoplasms \\
\hline 324.1 & Intraspinal abcess \\
\hline $730-730.99$ & Osteomyelitis \\
\hline $805.1,805.3,805.5,805.7,805.9$ & Open vertebral fractures \\
\hline $03.2-03.29$ & Chordotomy (procedure code) \\
\hline
\end{tabular}

To focus on patients with musculoskeletal back pain, we excluded patients with cancer, spinal infections, or open fractures. Including all patients with even a single index visit for back pain implies a mix of patients with acute, subacute, and chronic pain.

\section{Defining Episodes of Opioid Use}

For this purpose, we considered electronic pharmacy and medical record data for 6 months before and after an index visit (ie, including data from 2003 and 2005). This time interval was used with the recognition that, for many patients, the index visit in 2004 would not be their first visit because of back pain; a relapsing course is common for low back pain. We sought to identify opioid use that might precede or follow the index visit by a relatively short interval.

Using definitions from Von Korff et al, ${ }^{19}$ opioid use was defined as "none," "acute" ( $\leq 90$ days), "episodic," or "long term" ( $\geq 120$ days or $>90$ days with 10 or more fills). Episodic use was for $>90$ days but $<120$ days and with fewer than 10 fills of opioid medication. This was intended to identify opioid prescribing that was intermediate between acute and chronic use.

This classification considered cumulative opioid use during the year of observation, even though it might be discontinuous. Opioid use might represent single or multiple opioid preparations (eg, both a long-acting drug and a short-acting drug for "breakthrough" pain). We classified opioids as long- or short-acting and calculated morphine equivalents (Table 2). ${ }^{19}$

\section{Mental Health Diagnoses}

We searched medical records for 1 year before the index visit for any coded International Classification of Diseases, Ninth Revision, Clinical Modification diagnoses for depression (codes 296.2, 296.3, 300.4, 309.0, 309.1, 311); anxiety (codes 300.0300.09); posttraumatic stress disorder (code 
Table 2. Classification of Opioid Medications and Morphine Equivalent Conversion Factors per Milligram of Opioid*

\begin{tabular}{|c|c|c|}
\hline Major Group & Type of Opioid & $\begin{array}{l}\text { Morphine Equivalent } \\
\text { Conversion Factor/ } \\
\text { Milligram of Opioid }\end{array}$ \\
\hline \multirow[t]{7}{*}{ Short-acting, non-schedule II } & Propoxyphene (with or without aspirin/acetaminophen/ibuprofen) & 0.23 \\
\hline & Codeine + acetaminophen, ibuprofen, or aspirin & 0.15 \\
\hline & $\begin{array}{l}\text { Hydrocodone }+ \text { acetaminophen, ibuprofen, aspirin, or } \\
\text { homatropine }\end{array}$ & 1.00 \\
\hline & Tramadol with or without aspirin & 0.10 \\
\hline & $\begin{array}{l}\text { Butalbital and codeine (with or without aspirin, ibuprofen, } \\
\text { acetaminophen) }\end{array}$ & 0.15 \\
\hline & $\begin{array}{l}\text { Dihydrocodeine (with or without aspirin, ibuprofen, } \\
\text { acetaminophen) }\end{array}$ & 0.25 \\
\hline & Pentazocine (with or without aspirin, ibuprofen, acetaminophen) & 0.37 \\
\hline \multirow[t]{7}{*}{ Short-acting, schedule II } & Morphine sulfate & 1.00 \\
\hline & Codeine sulfate & 0.15 \\
\hline & Oxycodone (with or without aspirin, ibuprofen, acetaminophen) & 1.50 \\
\hline & Hydromorphone & 4.00 \\
\hline & Meperidine hydrochloride & 0.10 \\
\hline & Fentanyl citrate transmucosal ${ }^{\dagger}$ & 0.125 \\
\hline & Oxymorphone & 3.00 \\
\hline \multirow[t]{5}{*}{ Long-acting, schedule II } & Morphine sulfate sustained release & 1.00 \\
\hline & Fentanyl transdermal $^{\ddagger}$ & 2.40 \\
\hline & Levorphanol tartrate & 11.0 \\
\hline & Oxycodone $\mathrm{HCl}$ controlled release & 1.50 \\
\hline & Methadone & 3.00 \\
\hline
\end{tabular}

${ }^{*}$ Opioids delivered by pill, capsule, liquid, transdermal patch, and transmucosal administration were included. Opioids formulated for administration by injection or suppository were not included.

${ }^{\dagger}$ Transmucosal fentanyl conversion to morphine equivalents assumes $50 \%$ bioavailability of transmucosal fentanyl, and $100-\mu \mathrm{g}$ transmucosal fentanyl is equivalent to 12.5 to $15 \mathrm{mg}$ of oral morphine.

fTransdermal fentanyl conversion to morphine equivalent is based on the assumption that one patch delivers the dispensed micrograms/hour over a 24-hour day and remains in place for 3 days.

Adapted from Von Korff M, Saunders K, Ray GT, et al. De facto long-term opioid therapy for noncancer pain. Clin J Pain $2008 ; 24: 521-7 . .^{19}$

309.81); or substance abuse (codes 303.xx, 304.xx, 305.xx). The diagnoses were not based on standardized measures but on clinicians' judgments.

\section{Measures of Patient Comorbidity and Health Care Use}

We recorded patient demographics, comorbidity score, smoking, and body mass index. We also measured aspects of health care utilization, including co-prescription of sedative-hypnotics, emergency department visits, other clinic visits, and hospitalizations for the year surrounding the index date (6 months before and 6 months after).

Sedative-hypnotic drugs were those identified using the Medi-Span Generic Product Identifier ${ }^{20}$ or the American Hospital Formulary Service drug information compendium. ${ }^{21}$ These included benzodiazepines; barbiturates; so-called "z-drugs" (zol- pidem, eszopiclone, zaleplon); carisoprodol; and less frequently prescribed drugs such as diphenhydramine, chloral hydrate, and meprobamate.

Comorbidity was measured using the RxRisk score, a pharmacy-based model designed to predict future health care costs based on patient age, sex, Medicare or Medicaid insurance coverage, and use of drugs closely linked to specific chronic conditions (eg, biguanides, insulins, sulfonylureas for diabetes). ${ }^{22,23} \mathrm{~A}$ score is calculated from a regression model that weights each diagnosis according to its ability to predict future costs. RxRisk was developed in a managed care system with electronic records similar to KPNW. To calculate scores, we used records for the 1-year period before the index visit. For adults, the RxRisk calculation excludes analgesics because they are prescribed with too 
much discretion to be appropriate for a payment adjustment model. ${ }^{22}$ In addition, we considered the number of hospitalizations in the past year as a crude marker of illness burden.

\section{Analysis}

Because we hypothesized that certain lifestyle and mental health conditions would increase as duration of opioid use increased, we used the CochraneArmitage test for trend to compare proportions of patient characteristics, diagnoses, health care utilization, and prescription patterns across ordered categories of opioid use. This approach tests for an increasing or decreasing trend in patient characteristics and outcomes across ordered groups as opposed to testing for any differences in proportions.

For continuous data, we used the Kruskall-Wallis nonparametric rank-sum test or bivariate generalized linear models where appropriate. Multivariate analyses of dichotomous outcomes were conducted using logistic regression. For clinic visits, Poisson regression was used because of the non-normal count distribution. Each model adjusted for patient age, sex, and comorbidity score. Depending on the analysis and study question, some models also were adjusted for use of sedativehypnotics, number of hospitalizations, morphine dose at last dispensing before the index visit, and type of opioid (long or short acting). Analyses were conducted using SAS software (version 9.2, SAS Institute, Cary, NC), and utilized a two-tailed $P<$ .05 to define statistical significance.

\section{Results}

\section{Patients and Diagnoses}

There were 26,014 patients with a diagnosis of low back pain who met our eligibility criteria. Of these, $15,830(61 \%)$ received at least one opioid prescription during the year surrounding the index visit. Of those receiving opioids, 4883 (18.8\% of all patients with back pain) had an episode of long-term opioid use during that year.

Among all patients with back pain, mean age was 50.3 years (SD, 16.6), and $56.5 \%$ were women. Among those with a recorded race $(31 \%$ were missing), $89.3 \%$ were white, $3.2 \%$ were black, $2.5 \%$ were Asian, and $1.1 \%$ were American Indian or Alaska Native; 4\% self-reported other races. Among those reporting ethnicity (48\% missing), $4.6 \%$ were Hispanic.
Seventy-eight percent of patients received nonspecific diagnoses such as "low back pain" or "sprains and strains." Another 12\% had herniated discs, sciatica, degenerative discs, or spinal stenosis. The remainder received a variety of diagnoses, eg, spondylolisthesis and closed vertebral fractures.

The most frequently prescribed opioids were hydrocodone with acetaminophen; oxycodone with acetaminophen; acetaminophen with codeine; oxycodone $\mathrm{HCl}$, and morphine sulfate. These five preparations accounted for $92 \%$ of prescriptions.

\section{Patient Characteristics Associated With Opioid Use}

Table 3 divides the patients with back pain into four categories of opioid use: none, acute, episodic, or chronic. The table represents each patient only once according to his or her longest episode of opioid use. Mean patient age rose with increasing duration of opioid use, as did the proportion of women. The comorbidity score also increased with increasing duration of opioid use (all $P<.001$ ). Obesity and smoking were also associated with longer opioid use. Among long-term opioid users, $52.6 \%$ were current or recent smokers and $50.0 \%$ had a body mass index $\geq 30(P<.001)$.

\section{Mental Health Diagnoses}

Diagnoses of depression, anxiety, and substance abuse increased in a monotonic trend across categories of increasing duration of opioid use $(P<$ .001 ; Figure 1 and Table 3). Among long-term opioid users, $31 \%$ had a diagnosis of depression, and $49 \%$ had at least one of these four mental health diagnoses. However, even patients with acute opioid use had a higher prevalence of depression than patients who had back pain with no opioid use (17.4\% vs $12.2 \%)$. This was true for each of the mental health diagnoses.

All the associations between mental health diagnoses and duration of opioid use remained statistically significant after adjusting for patient age, sex, comorbidity, hospitalizations, and (in some cases) sedative-hypnotic use in logistic regression models (Table 4). For depression and substance abuse- the most common of these diagnoses-the odds ratios showed a monotonic pattern of association with duration of opioid use. 
Table 3. Kaiser Northwest Patient Demographic Characteristics and Comorbid Conditions According to Duration of Opioid Use, 2004*

\begin{tabular}{|c|c|c|c|c|c|}
\hline Characteristic & No opioids & Acute opioids only ${ }^{\dagger}$ & Episodic opioid use $^{\dagger}$ & Chronic opioid use $^{\dagger}$ & $P$ \\
\hline Patients (n) & 10,184 & 10,543 & 404 & 4,883 & - \\
\hline Mean age (years) ${ }^{\ddagger}$ & 49.1 & 49.1 & 56.6 & 54.6 & $<.001$ \\
\hline Women ${ }^{\S}$ & $5,529(54.3)$ & $5,847(55.5)$ & $240(59.4)$ & $3,071(62.9)$ & $<.001$ \\
\hline $\mathrm{BMI} \geq 30^{\S}$ & $3,490(36.8)$ & $4,525(45.4)$ & $185(47.2)$ & $2,403(50.0)$ & $<.001$ \\
\hline Current or recent smoker ${ }^{\S}$ & $3,538(37.4)$ & $4,620(45.9)$ & $176(46.3)$ & $2,476(52.6)$ & $<.001$ \\
\hline \multicolumn{6}{|l|}{ Diagnosis during previous year } \\
\hline Depression $^{\S}$ & $1,247(12.2)$ & $1,839(17.4)$ & $95(23.5)$ & $1,526(31.3)$ & $<.001$ \\
\hline Anxiety ${ }^{\S}$ & $449(4.4)$ & $594(5.6)$ & $25(6.2)$ & $573(11.7)$ & $<.001$ \\
\hline $\mathrm{PTSD}^{\S}$ & $54(0.5)$ & $102(1.0)$ & $1(0.3)$ & $116(2.4)$ & $<.001$ \\
\hline Substance abuse $\mathrm{S}^{\S}$ & $946(9.3)$ & $1,467(13.9)$ & $60(14.9)$ & $1,233(25.3)$ & $<.001$ \\
\hline $\begin{array}{l}\text { Patients with any of the four mental } \\
\text { health diagnoses } \$\end{array}$ & $2,185(21.5)$ & $3,108(29.5)$ & $146(36.1)$ & $2,405(49.3)$ & $<.001$ \\
\hline Median comorbidity score (RxRisk) ${ }^{\mathbb{I}}$ & 1,276 & 1,580 & 2,464 & 3,366 & $<.001$ \\
\hline
\end{tabular}

Values provided as $\mathrm{n}(\%)$ unless otherwise indicated.

*Approximately $5 \%$ of patients had missing values for body mass index and smoking status; the percentages displayed are calculated for those cases that were not missing.

†"Acute" use was defined as use $\geq 90$ days. "Episodic" use was defined as use $>90$ days but $<120$ days with fewer than 10 prescription fills. "Chronic" use was $\geq 120$ days, or $>90$ days with $\geq 10$ prescription fills.

${ }^{\ddagger}$ Generalized linear model F-test.

${ }^{\S}$ Cochrane-Armitage test for trend.

"Kruskal-Wallis test.

BMI, body mass index; PTSD, posttraumatic stress disorder.

\section{Health Care Use and Co-Prescribing of Sedative Hypnotics}

Greater sedative-hypnotic use was associated with longer opioid use in an incremental fashion, ranging from $10 \%$ among nonopioid users to $44.4 \%$ among chronic opioid users (Figure 1; Table 5). This association persisted in a multivariate model adjusted for age, sex, comorbidity, and number of hospitalizations. For example, the odds ratio for sedative-hypnotic use was 4.0 (95\% CI, 3.65-4.39) for chronic opioid use compared with no opioid use (Table 4). Benzodiazepines were the most commonly prescribed sedative-hypnotics; diazepam, lorazepam, alprazolam, clonazepam, oxazepam, and temazepam accounted for $80 \%$ of prescriptions. Adding hydroxyzine and zolpidem accounted for $94 \%$ of sedative-hypnotic prescriptions.

Almost $60 \%$ of long-term opioid users received only short-acting opioids. Among the $40 \%$ who received long-acting opioids, nearly all received both long- and short-acting opioids (Table 6). Among those with acute opioid use, 98\% received only short-acting drugs.

Just more than $36 \%$ of those with any opioid use had an emergency room visit during the study year, and approximately one third of these were associ- ated with a back pain diagnosis (Table 5). An opioid prescription was filled within 5 days after $56 \%$ of emergency room visits. These percentages were similar among patients with any duration of opioid use. Emergency room use remained associated with opioid use after adjustment for age, sex, comorbidity, number of hospitalizations, and sedative-hypnotic use. In this case, odds ratios for any duration of opioid use were similar and clustered around 2.0 (chronic opioid use compared with no opioid use: odds ratio, 1.85; 95\% CI, 1.69-2.02).

These patients with back pain made heavy use of other clinic services. The median number of all clinic visits during the year surrounding the index date was 18 for patients receiving chronic opioids, 8 for those not using opioids, and intermediate for those with acute or episodic opioid use (Table 5). In a Poisson regression, the trend for increasing clinic use remained associated with greater duration of opioid use. For example, even after adjustment for age, sex, comorbidity, and number of hospitalizations, patients receiving long-term opioids had a $41 \%$ higher rate of clinic visits than patients with no opioid use (relative risk, 1.41 ; $95 \%$ CI, $1.37-$ 1.45). The number of different opioid prescribers also increased with increasing duration of opioid 
Figure 1. Graphic presentation of proportions of patients with diagnoses of depression (a), any of four mental health diagnoses (b), or sedative hypnotic use (c) as a function of duration of opioid use. PTSD, posttraumatic stress disorder.
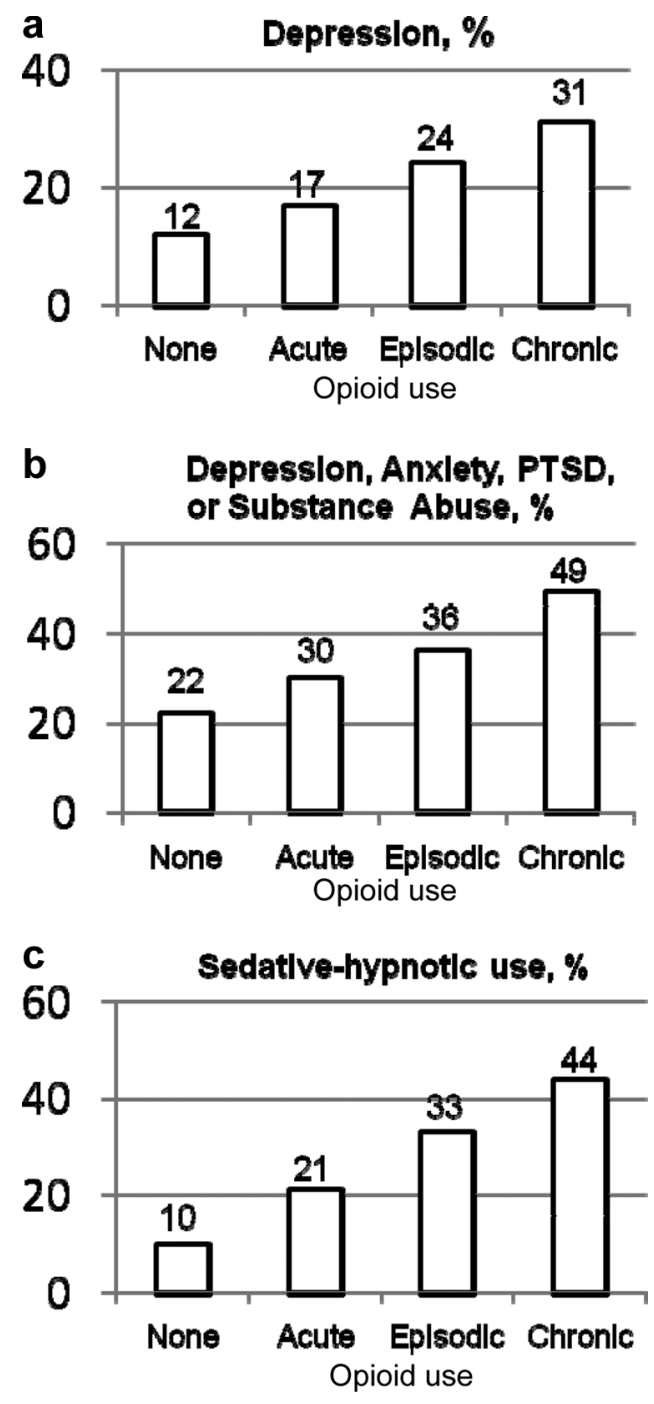

use (Table 5). Most patients were not seen in a specialty pain clinic; only $12 \%$ of even chronic opioid users had such a visit during the year surrounding the index visit.

Although only $3.6 \%$ of nonopioid users were hospitalized during this time, more than $20 \%$ of episodic or long-term opioid users were hospitalized (Table 5). In logistic regression models, the high risk of hospitalization among episodic and chronic opioid users persisted even after adjustment for age, sex, comorbidity score, and use of sedative-hypnotics. The odds ratio among those with acute use of opioids was 2.89 (95\% CI, 2.55-
$3.28)$, for episodic use the odds ratio was 4.70 (95\% CI, 3.57-6.19), and for chronic use the odds ratio was 3.90 (95\% CI, 3.40-4.47).

\section{Discussion}

Opioid prescribing was common among patients with back pain, and almost $20 \%$ received long-term opioids. Increasing duration of opioid use was associated with increasing age and comorbidity, suggesting that some opioid prescribing was related to conditions other than back pain or in addition to back pain. Our data suggest that patients with back pain have a substantial comorbidity load that is associated with the duration of opioid use. Using data from development of the RxRisk score, ${ }^{22}$ we can estimate that the median RxRisk score of our patients receiving chronic opioid therapy was similar to that of age- and sex-matched peers with coronary or peripheral vascular disease. The median score of our "no opioid" group is about the same as that of patients with hypertension.

Increasing duration of opioid use was strongly associated with an increasing prevalence of mental health conditions (depression, anxiety, posttraumatic stress disorder, or substance abuse); almost $50 \%$ of patients receiving long-term opioids had at least one of these diagnoses. Similarly, negative health habits (obesity, smoking) were associated with duration of opioid use. The rate of smoking among long-term opioid users was particularly high (more than 50\%).

Our data confirm other observations regarding mental health and chronic opioid use. ${ }^{11,12}$ However, our findings indicate that this association is not confined to long-term opioid users in some threshold fashion, but that it is found in incremental fashion among increasing durations of opioid use. The causality of these associations is unclear. In a study of sequential surveys, depression and anxiety at the first survey predicted a higher likelihood of opioid use at the second survey 3 years later. $^{24}$ Thus, it seems plausible that depression leads to more opioid use, though it also may be true that opioid use results in greater depression or that both of these conditions are associated with some confounding factor. Etiology aside, these associations may be of interest if they are merely predictive of long-term opioid use.

The wisdom of long-acting opioid use for chronic pain remains controversial, but is based on 
Table 4. Logistic Regression Models for the Associations of Mental Health Diagnoses With Duration of Prescription Opioid Use*

\begin{tabular}{lccccc}
\hline & Depression & Anxiety & PTSD & Substance Abuse & Sedative-Hypnotic Use \\
\hline None (reference category) & 1.00 & 1.00 & 1.00 & 1.00 & 1.00 \\
Acute $^{\dagger}$ & $1.15(1.06-1.25)$ & $1.00(0.88-1.14)$ & $1.34(0.96-1.88)$ & $1.44(1.32-1.57)$ & $1.94(1.79-2.11)$ \\
Episodic $^{\dagger}$ & $1.35(1.05-1.74)$ & $0.95(0.62-1.45)$ & $0.31(0.04-2.28)$ & $1.56(1.17-2.08)$ & $2.99(2.38-3.75)$ \\
Chronic $^{\dagger}$ & $1.49(1.35-1.64)$ & $1.44(1.24-1.66)$ & $2.07(1.44-2.96)$ & $2.77(2.50-3.08)$ & $4.00(3.65-4.39)$ \\
\hline
\end{tabular}

Values provided as odds ratios $(95 \% \mathrm{CI})$.

*All models adjusted for age, gender, comorbidity score, and number of hospitalizations in the past year. The models for depression and for substance abuse also adjusted for the use of sedative-hypnotics.

‘"Acute" was defined as use for $\geq 90$ days. "Episodic" was defined as use for $>90$ days but $<120$ days with fewer than 10 prescription fills. "Chronic" was defined as use for $\geq 120$ days or $>90$ days with 10 or more prescription fills.

PTSD, posttraumatic stress disorder.

theoretical considerations of more consistent pain control and more normal sleep patterns, assuming dosing is less frequent. ${ }^{25}$ These considerations led to a KPNW guideline recommending long-acting drug use for chronic pain, and KPNW used this as a quality indicator during the study period. Although use of long-acting opioids increased with duration of use, the frequent use $(59 \%)$ of shortacting opioids among patients receiving long-term therapy supports Von Korff et al's ${ }^{19}$ concept of de facto long-term prescribing. That is, many patients may become long-term opioid users through clinical inertia: clinicians continue to refill prescriptions for short-acting opioids and do not consider a deliberate decision for long-term use with informed consent and an opioid agreement.

We do not know the extent to which clinicians cautioned patients against concurrent use of sedative-hypnotics and opiates, but their co-prescribing presents a potential safety concern. Sedative-hyp-

Table 5. Health Care Use and Complications According To Duration of Opioid Use

\begin{tabular}{|c|c|c|c|c|c|}
\hline Type of Health Care Use & No Opioids & Acute Opioids Only & Episodic Opioid Use & Chronic Opioid Use & $P$ \\
\hline Patients (n) & 10,184 & 10,543 & 404 & 4,883 & \\
\hline $\begin{array}{l}\text { Opioid dose at last dispensing, morphine } \\
\text { equivalent (median)* }\end{array}$ & $\mathrm{NA}^{\ddagger}$ & $30.0 \mathrm{mg}$ & $20.0 \mathrm{mg}$ & $30.0 \mathrm{mg}$ & $<.001$ \\
\hline Opioid prescribers (median) ${ }^{*}$ & $\mathrm{NA}^{\ddagger}$ & 1 & 2 & 3 & $<.001$ \\
\hline $\begin{array}{l}\text { Patients receiving sedative-hypnotic } \\
\text { prescription during the } 6 \text { months } \\
\text { before/after index visit }(\mathrm{n}[\%])^{\dagger}\end{array}$ & $1,018(10.0)$ & $2,163(20.5)$ & $134(33.2)$ & $2,166(44.4)$ & $<.001$ \\
\hline $\begin{array}{l}\text { Patients with ER visit } 6 \text { months before/ } \\
\text { after index date }(\mathrm{n}[\%])^{\dagger}\end{array}$ & $1,725(16.9)$ & $3,627(34.4)$ & $148(36.6)$ & $1,948(39.9)$ & $<.001$ \\
\hline $\begin{array}{l}\text { ER visit with back pain diagnosis, } \\
\text { patients with any ER visit }(\mathrm{n}[\%])^{\dagger}\end{array}$ & $405(23.5)$ & $1,246(34.4)$ & $50(33.8)$ & $535(27.5)$ & .66 \\
\hline $\begin{array}{l}\text { Opioid prescription filled within } 5 \text { days } \\
\text { of ER visit (\% of patients with ER } \\
\text { visit) }\end{array}$ & $1(0.1)$ & $2,048(56.5)$ & $85(57.4)$ & $1,091(56.0)$ & $<.001$ \\
\hline $\begin{array}{l}\text { Clinic visits of any type during the } 6 \\
\text { months before/after index } \\
\text { date (median) }\end{array}$ & 8 & 11 & 17 & 18 & $<.001$ \\
\hline $\begin{array}{l}\text { Patients with any pain clinic visit during } \\
\text { the } 6 \text { months before/after index } \\
\text { date }(\mathrm{n}[\%])^{\dagger}\end{array}$ & $99(1.0)$ & $227(2.2)$ & $25(6.2)$ & $585(12.0)$ & $<.001$ \\
\hline $\begin{array}{l}\text { Patients with any hospitalization during } \\
\text { the } 6 \text { months before or after index } \\
\text { date }(\mathrm{n}[\%])^{\dagger}\end{array}$ & $364(3.6)$ & $1,126(10.7)$ & $87(21.5)$ & $1,012(20.7)$ & $<.001$ \\
\hline
\end{tabular}

${ }^{* a}$ Kruskal-Wallis test.

${ }^{\dagger}$ Cochrane-Armitage test for trend.

${ }^{\ddagger} \mathrm{NA}$, not applicable. This category was not included in tests of statistical significance.

ER, emergency room. 
Table 6. Use of Long-and Short-Acting Opioids by Duration of Opioid Use

\begin{tabular}{lccr}
\hline Opioid & $\begin{array}{c}\text { Acute Use } \\
(\mathrm{n}=10,543)\end{array}$ & $\begin{array}{c}\text { Episodic Use } \\
(\mathrm{n}=404)\end{array}$ & $\begin{array}{r}\text { Chronic Use } \\
(\mathrm{n}=4,883)\end{array}$ \\
\hline Short-acting only (\%) & 98.3 & 85.2 & 59.4 \\
Long-acting only (\%) & 0.1 & 0.7 & 1.3 \\
Both long-and short-acting (\%) & 1.6 & 14.1 & 39.3 \\
\hline
\end{tabular}

notics in combination with opioids may increase the risk of oversedation, overdose, and mortality. ${ }^{6,17}$ Furthermore, benzodiazepine use may predict subsequent use of opioids. ${ }^{26}$ The greater use of sedative-hypnotics with longer-term opioid use is contrary to the expectation based on expert recommendations, but it also has been observed in other health care systems. ${ }^{14,27,28}$ It is unclear if the high use of sedative-hypnotics among long-term opioid users is a result of pre-existing anxiety or sleep disorders, sleep disorders induced by opioids, or a manifestation of "addictive personalities." Some of these drugs-specifically diazepam-may have been used for muscle relaxant actions, suggesting an opportunity for clinician education.

Emergency department use was high among opioid users of any duration, though a minority of visits was associated with back pain. Nonetheless, more than half the emergency room visits were associated with a prescription for opioid medication. These patients also were heavy users of all clinic services. Some use is likely associated with monitoring and refills of opioids, but comorbidity and mental health concerns also may be important. As with clinic visits, the probability of hospitalization was associated with opioid use. Episodic or chronic use was associated with nearly 4 -fold odds of being hospitalized, even after adjusting for demographics, comorbidity, and sedative-hypnotic use. A minority of patients were seen at a specialty pain clinic, suggesting that most, even with chronic opioid use, are managed in primary or emergency care. This was true even though access to pain clinic care was available in the form of pain management group visits throughout the practice region.

The number of different opioid prescribers increased with increasing duration of opioid use. This may suggest inadequate continuity of prescribing clinicians, but it also may be an inevitable consequence of long-term use. For example, if a patient's prescribing clinician is unavailable when medication supplies run low, the patient's immediate needs would be met by other available clinicians (eg, "doctor of the day").

\section{Strengths and Limitations}

Our data have the advantage of representing a large population, many providers, and nearly complete capture of health care utilization. However, there are important limitations. The study population represents the racial/ethnic mix in the Portland metropolitan area but underrepresents minority populations. Thus, the results must be generalized with caution.

Although every patient had back pain, we do not know the original indication for prescribing opioids. Clinical experience suggests that this often may be unclear for long-term opioid users, even when the full medical record is examined. Furthermore, many patients have multiple pain conditions, and it may be misleading to single out one diagnosis. $^{28}$

We cannot know the degree to which the associations we found are causal or the direction of any causation. However, the graded association of many variables with the duration of opioid use strengthens an argument for causal associations. These findings may be useful for risk management, even if they merely predict rather than have an etiologic role.

In the case of diagnosed substance abuse, we cannot determine if these diagnoses are related to the prescribed opioids, other drugs, or both. To the extent that clinicians may use these diagnosis codes to indicate dependence on long-term prescribed opioids, they may be overused and misleading. Most patients with more than a few weeks of opioid therapy will experience withdrawal symptoms if opioids are discontinued (commonly referred to as "dependence"). This is distinct from the compulsive and aberrant drug use of addiction, though this distinction is not always clearly made, and we cannot know from these data how the diagnosis of "substance abuse" was used. 
From pharmacy data alone, we cannot know if patients were receiving opioids from other sources, including illicit use. Many states have a statewide Prescription Monitoring Program that collects dispensing data from all pharmacies and helps to identify potential misuse, but such a system is not yet operational in Oregon. We did not assess whether opioid doses were increasing or decreasing during an episode of use. Our data are from 2004 and may not fully reflect current practice, but they establish a baseline against which future findings can be measured.

\section{Conclusions}

These data suggest directions for improving the safety of opioid prescribing. The indications for combined opioid and sedative-hypnotic therapy should be scrutinized. Such co-prescribing has already become a target of quality improvement in some primary care practices. ${ }^{29}$ This concern might be part of an agenda for patient-physician shared decision making before embarking on long-term opioid use.

Redoubling efforts to ensure prescriber continuity for long-term opioid users; to monitor the interaction of comorbid conditions and medications; and to reduce use of clinic, emergency department, and hospital care also may be important. If many visits are motivated by patients seeking their next prescriptions-rather than addressing clinical complaints-system interventions or alternative treatment approaches may be warranted. The reasons for high clinic and hospital utilization deserve closer investigation to determine other strategies for mitigating such use. Because opioid prescribing is increasing most rapidly among patients with mental health and substance abuse diagnoses, ${ }^{11}$ primary care physicians may need to become more vigilant in screening for these conditions and addressing them before initiating opioid therapy.

Finally, these data suggest strategies for using electronic medical records to identify patients at high risk for long-term opioid use, drug misuse, or drug complications and to support clinical decision making. Such patterns may be unapparent when examining individual paper records. Others have begun to develop software to identify possible misuse or unsafe practices, ${ }^{30}$ and some decision aids are already being implemented by KPNW. This may represent a modern version of the approach advocated by Fry, ${ }^{31}$ who recommended examining one's own practice population to identify patterns of illness and care that could lead to improvements in care.

\section{References}

1. Hudson TJ, Edlund MJ, Steffick DE, Tripathi SP, Sullivan MD. Epidemiology of regular prescribed opioid use: results from a national, population-based survey. J Pain Sympt Mgmt 2008;36:280-8.

2. Eriksen J, Sjogren P, Bruera E, Ekholm O, Rasmussen NK. Critical issues on opioids in chronic noncancer pain: an epidemiological study. Pain 2006; 125:172-9.

3. Martell BA, O'Connor PG, Kerns RD, et al. Systematic review: opioid treatment for chronic back pain: prevalence, efficacy, and association with addiction. Ann Intern Med 207;146:116-27.

4. Caudill-Slosberg MA, Schwartz LM, Woloshin S. Office visits and analgesic prescriptions for musculoskeletal pain in US: 1980 vs. 2000. Pain 2004;109: 514-9.

5. Martin BI, Deyo RA, Mirza SK, et al. Expenditures and health status among adults with back and neck problems. JAMA 2008;299:656-64.

6. Franklin GM, Mai J, Wickizer T, Turner JA, FultonKehoe D, Grant L. Opioid dosing trends and mortality in Washington State workers' compensation, 19962002. Am J Ind Med 2005;48(2):91-9.

7. Luo X, Pietrobon R, Hey L. Patterns and trends in opioid use among individuals with back pain in the United States. Spine 2004;29:884-91.

8. Zerzan JT, Morden NE, Soumerai S, et al. Trends and geographic variation of opiate medication use in state Medicaid fee-for-service programs, 1996 to 2002. Med Care 2006;44:1005-10.

9. Compton WM, Volkow ND. Major increases in opioid analgesic abuse in the United States: concerns and strategies. Drug Alcohol Depend 2006;81: $103-7$.

10. Paulozzi LJ, Budnitz DS, Xi Y. Increasing deaths from opioid analgesics in the United States. Pharmacoepidemiol Drug Saf 2006;15:618-27.

11. Edlund MJ, Martin BC, Devries A, Fan M-Y, Braden JB, Sullivan MD. Trends in use of opioids for chronic noncancer pain among individuals with mental health and substance use disorders: the TROUP Study. Clin J Pain 2010;26:1-8.

12. Edlund MJ, Steffick D, Hudson T, Harris KM, Sullivan M. Risk factors for clinically recognized opioid abuse and dependence among veterans using opioids for chronic non-cancer pain. Pain 2007;129:355-62.

13. Wasan AD, Davar G, Jamison R. The association between negative affect and opioid analgesia in patients with discogenic low back pain. Pain 2005;117: $450-61$. 
14. Hermos JA, Young MM, Gagnon DR, Fiore LD. Characterizations of long-term oxycodone/acetaminophen prescriptions in veteran patients. Arch Intern Med 2004;164:2361-6.

15. Tetrault JM, Desai RA, Becker WC, Fiellin DA, Concato J, Sullivan LE. Gender and non-medical use of prescription opioids: results from a national US survey. Addiction 2007;103:258-68.

16. Stover BD, Turner JA, Franklin G, et al. Factors associated with early opioid prescription among workers with low back injuries. J Pain 2006;7:718-25.

17. Dunn KM, Saunders KW, Rutter CM, et al. Opioid prescriptions for chronic pain and overdose: a cohort study. Ann Intern Med 2010;152:85-92.

18. Cherkin DC, Deyo RA, Volinn E, Loeser JD. Use of the International Classification of Diseases (ICD-9$\mathrm{CM})$ to identify hospitalizations for mechanical low back problems in administrative databases. Spine 1992;17:817-25.

19. Von Korff M, Saunders K, Ray GT, et al. De facto long-term opioid therapy for noncancer pain. Clin J Pain 2008;24:521-7.

20. WoltersKluwer Health, Medi-Span. Master drug data base v2.5. Available at: http://www.medi-span. com/master-drug-database.aspx. Accessed 15 July 2010.

21. American Society of Health-System Pharmacists. AHFS drug information. Available at: http:// www.ahfsdruginformation.com/products_services/di_ ahfs.aspx. Accessed 15 July 2010.

22. Fishman PA, Goodman MJ, Hornbrook MC, et al. Risk adjustment using automated ambulatory pharmacy data: the RxRisk Model. Med Care 2003;41: 84-99.
23. Farley FJ, Harley CR, Devine JW. A comparison of comorbidity measurements to predict healthcare expenditures. Am J Manag Care 2006;12:110-7.

24. Sullivan MD, Edlund MJ, Zhang L, Unutzer J, Wells KB. Association between mental health disorders, problem drug use, and regular prescription opioid use. Arch Intern Med 2006;166:2087-93.

25. Kalso E, Allan L, Dobrogowski J, et al. Do strong opioids have a role in the early management of back pain? Recommendations from a European expert panel. Curr Med Res Opin 2005;21:1819-28.

26. Skurtveit S, Furu K, Bramness J, Selmer R, Tverdal A. Benzodiazepines predict use of opioids-a follow-up study of 17,074 men and women. Pain Med 2010;11:805-14.

27. Morasco BJ, Duckart JP, Carr TP, Deyo RA, Dobscha SK. Clinical characteristics of veterans prescribed high doses of opioid medications for chronic non-cancer pain. Pain 2010;151:625-32.

28. Boudreau D, von Korff M, Rutter CM, et al. Trends in long-term opioid therapy for chronic non-cancer pain. Pharmocoepidemiol Drug Saf 2009;18:1166-75.

29. Hooper S, Bruno R, Sharpe M, Tahmindjis A. Alprazolam prescribing in Tasmania: a two-fold intervention designed to reduce inappropriate prescribing and concomitant opiate prescription. Australas Psychiatry 2009;17:300-5.

30. Parente ST, Kim SS, Finch MD, et al. Identifying controlled substance patterns of utilization requiring evaluation using administrative claims data. Am J Manag Care 2004;10:783-90.

31. Fry J. Common Diseases: their nature, incidence, and care. 4th edition. Lancaster: MTP Press Limited; 1985:9-10. 